\title{
Muscle endurance measurement using a progressive workload and a constant workload by maximal voluntary contraction
}

\author{
Shinichi Demura ${ }^{1}$, Masakatsu Nakada ${ }^{2 *}$ \\ ${ }^{1}$ Graduate School of Natural Science \& Technology, Kanazawa University, Kanazawa, Japan; \\ ${ }^{2}$ National Defense Academy, Yokosuka, Japan; *Corresponding Author: nakada@nda.ac.jp.
}

Received 12 August 2010; revised 18 August 2010; accepted 25 August 2010.

\begin{abstract}
Muscle endurance measurement using a progressive workload method may reduce pain sensation in the subject. This study aimed to examine the relationships between force-time parameters during sustained static gripping as measured by maximal voluntary contraction (MVC) using either a progressive workload (PW) or a constant workload (CW). Sixteen subjects performed sustained static gripping with 7 gradually increasing relative demand values of $20 \%$ to $80 \%$ MVC and sustained static gripping by MVC. The staging of progressive workload was $10 \mathrm{~s}$ for $20 \%$ MVC, $20 \mathrm{~s}$ each for $30,40,50,60$, and $70 \% \mathrm{MVC}$, and $10 \mathrm{~s}$ for $80 \% \mathrm{MVC}$. The forces exerted at $120 \mathrm{~s}$ in the $\mathrm{CW}$ and $\mathrm{PW}$ methods were at around the $23-27 \%$ MVC level. Peak force, final force, and force during the last $30 \mathrm{~s}$ for the PW method evaluated muscle endurance after $1 \mathrm{~min}$ and showed high correlations ( $r=$ $0.746-0.895)$. Significant correlations $(r=0.575$ -0.605 ) were found between time to $40 \%$ MVC in the CW method and peak force, final force, and force in the last $30 \mathrm{~s}$ in the PW method group. The peak force in the PW method may be useful for evaluating muscle endurance with a short testing time and without high pain sensation.
\end{abstract}

Keywords: Sustained Static Gripping; Progressive Workload; Constant Workload; Muscle Endurance

\section{INTRODUCTION}

Muscle endurance has been measured by maximal voluntary contraction (MVC) or fixed relative load intensity [1-6]. Many researchers have reported force-decrease properties exerted in the constant workload (CW) me- thod [6-8]. Yamaji et al. [8] examined the physiological properties of force-time parameters during maximal sustained static gripping and reported that time to $40 \%$ MVC reflects the individual difference of force based on oxygen debt with muscle blood flow limitation. Muscle endurance measurement using the force exertion by MVC may be effective, but there are problems with severe muscle-fatigue or dislike of the testing procedure. In the case of evaluating muscle endurance from the perspective of sustaining demand values, according to Nagasawa et al. [6], the sustained time is very short (about $16 \pm 13 \mathrm{sec}$ ) when using the heavy workload (75\% MVC) and is very long (about $224 \pm 144 \mathrm{sec}$ ) when using the light workload (25\% MVC). Even when using any workload, the force value reaches a steady state at $15 \%-20 \% \mathrm{MVC}$ and then decreases little $[6,8,9]$.

Meanwhile, the progressive workload (PW) method gradually increases the relative loads while considering the physiological responses $[10,11]$. Measuring muscle endurance by this method can reduce the physical burden on the subjects (dislike, pain sensation, etc), because this method does not impose rapidly large workloads on the muscle groups. The PW method is also considered to be effective for the elderly, with whom there is increased risk associated with a rapid increase of blood pressure.

When considering the physiological parameters of muscle endurance measurements based on MVC, it is important to remember that during the initial force exertion due to large workloads, muscle endurance is evaluated in a state of blood flow obstruction caused by an increase in intramuscular pressure. On the other hand, the low force exertion state in the latter half of the measurement period evaluates muscle endurance in a state of sufficient oxygen delivery to the muscles, with the resumption of blood flow caused by the reduction of intramuscular pressure $[8,12]$. The PW method may be able to evaluate muscle endurance without significant fatigue or dislike 
and with higher safety. However, the relationships between evaluation parameters of muscle endurance for the PW method and for the constant workload (CW) method are unclear. It is assumed that the parameters in above both methods have close relationships, because they are measuring the same grip muscle endurance.

This study aimed to examine the relationships between force-time parameters during sustained static gripping using both a progressive workload and a constant workload by maximal voluntary contraction.

\section{METHODS}

\subsection{Subjects}

Subjects were 16 young male adults (height $172.7 \pm$ $5.2 \mathrm{~cm}$, body weight $67.1 \pm 6.1 \mathrm{~kg}$, and age $21.6 \pm 2.0$ years). Written informed consent was obtained from all subjects after a full explanation of the experimental purpose and protocol.

\subsection{Materials}

Grip strength was measured using a digital hand dynamometer with a load-cell sensor (EG-100, Sakai, Japan). Each signal was sampled at $20 \mathrm{~Hz}$ through an analog-to-digital interface and then relayed to a personal computer. The changes of force values on the computer display were shown on a time-series graph on the horizontal scale, and relative values were shown on the vertical scale. The display of the target force line was fed back to the subjects.

\subsection{Setting of Progressive and Constant Workloads}

When using loads over $75 \%$ maximum voluntary contraction (MVC), subject can sustain a target force $[6,13]$. In addition, the force exertion value in loads of less than $20 \%$ of MVC decreases little [6]. Hence, this study selected a measurement time of 2 min with progressive workloads of 20\%-80\% MVC. Yamaji et al. [14] reported that the gripping force during maximal sustained hand grip remarkably decreased until about 30-60 s and reached an almost steady state when it decreased to $20 \%$ MVC (within about $150 \mathrm{~s}$ ). Hence, a constant workload of $100 \%$ MVC and a measurement time of 3 min were selected.

\subsection{Experimental Procedure}

After measuring maximal grip force, each subject performed the sustained static gripping using the dominant hand with a progressive workload having 7 relative de- mand values (20\% to $80 \%$ MVC) increasing by $10 \%$ MVC each. The subject's dominant hand was determined based on Oldfield's handedness inventory [15]. The time of the demand values was $10 \mathrm{sec}$ for $20 \% \mathrm{MVC}, 20 \mathrm{sec}$ each for 30, 40, 50, 60, and 70\% MVC, and $10 \mathrm{sec}$ for $80 \%$ MVC. The sustained static maximal griping time was $3 \mathrm{~min}$ for $100 \%$ MVC. Considering the fatigue effect, each measurement was performed once a day.

\subsection{Parameters}

Referring to previous studies [8,9] using sustained static maximal hand gripping, the following progressive workload (PW) parameters were selected: 1) peak of force value (peak force), 2) time of peak force (peak force time), 3) final force value (final force), and 4) average force during the last $30 \mathrm{~s}$ (last $30 \mathrm{~s}$ force). The final force value was the force exerted at $120 \mathrm{~s}$ (Figure 1). The following constant workload (CW) parameters by maximal voluntary contraction were selected: the time required to decrease from maximal grip strength to 1) $30 \% \mathrm{MVC}$, 2) 40\% MVC, 3) 60\% MVC, and 4) $80 \%$ $\mathrm{MVC}$; 5) $120 \mathrm{~s}$ force; and 6) final force value (final force) (Figure 2).

\subsection{Data Analysis}

Pearson's correlation coefficient was used to examine the relationships between $\mathrm{CW}$ parameters and $\mathrm{PW}$ parameters. A probability level of 0.05 was used as indicative of statistical significance.

\section{RESULTS}

Figures 3 and 4 show average curves of changes in time-series forces during sustained static gripping by MVC using either PW or CW. The peak force appeared at $60 \%$ MVC. The force then decreased until the end of the $2 \mathrm{~min}$ measurement, and the final force was about $27 \%$ MVC. The individual difference of force exertion

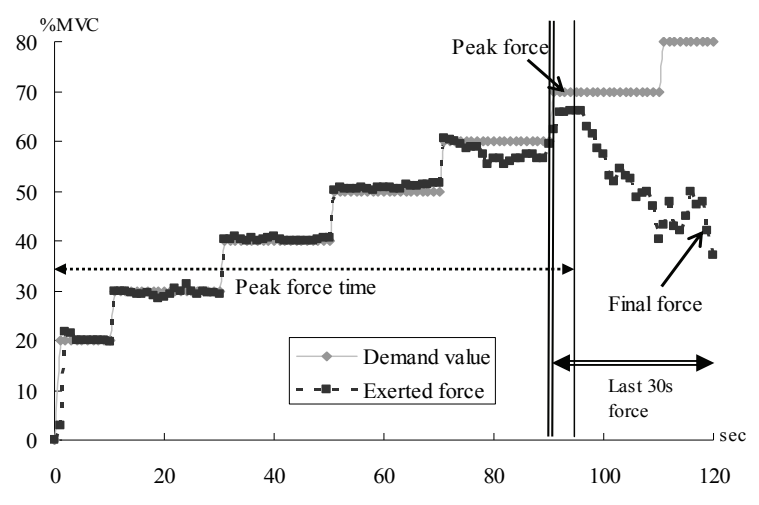

Figure 1. Progressive workload parameter. 


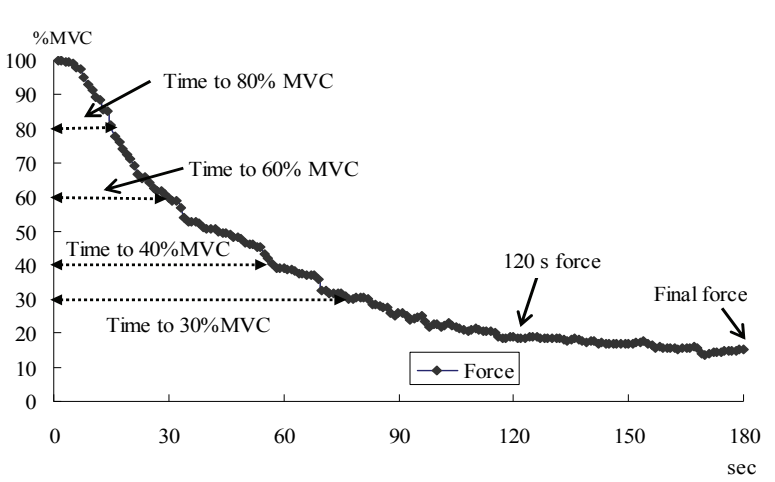

Figure 2. Constant workload parameter.

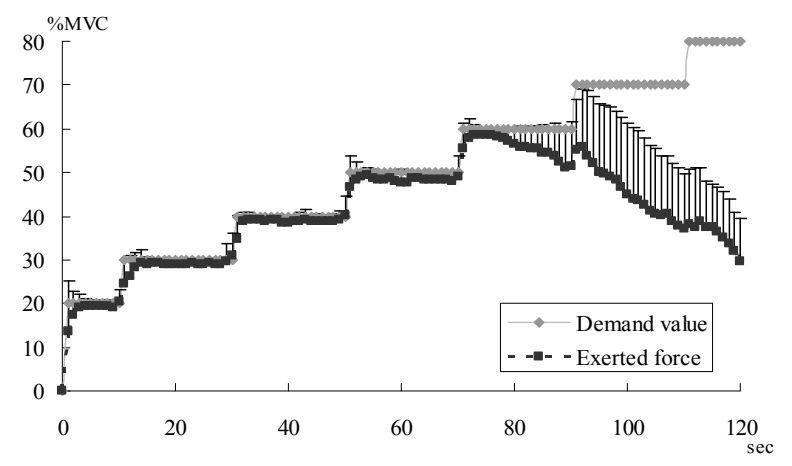

Figure 3. Average curves of changes in time-series forces during sustained static gripping by MVC using either PW.



Figure 4. Average curves of changes in time-series forces during sustained static gripping by MVC using either CW.

values increased from $60 \mathrm{~s}$ (demand value: $50 \%$ MVC) to $90 \mathrm{~s}$ (demand value: $70 \% \mathrm{MVC}$ ) after the onset of sustained static gripping. The force values during sustained static gripping using MVC decreased markedly until $60 \mathrm{~s}$ after sustained static grip onset and reached nearly $40 \%$ MVC. The individual difference of force values increased until $30 \mathrm{~s}$ after the onset of gripping and then reduced until $60 \mathrm{~s}$ at which point a steady state was reached.

Table 1 shows the correlations between PW and CW parameters. Significant and high correlations $(r=0.713$ 0.895) were found among PW parameters. In CW parameters, significant and high correlations $(\mathrm{r}=0.709$ 0.868 ) were found between time to $80 \% \mathrm{MVC}$ and time to $60 \% \mathrm{MVC}$, between time to $60 \% \mathrm{MVC}$ and time to $40 \% \mathrm{MVC}$, and between times to $30 \%-40 \% \mathrm{MVC}$ and $120 \mathrm{~s}$ force. Time to $40 \% \mathrm{MVC}$ in the $\mathrm{CW}$ method showed significant correlations with peak force, final force, and last $30 \mathrm{~s}$ force in the PW method.

Mean of peak force time in the PW method was about $83 \mathrm{~s}$, and mean decreased times until $80 \%, 60 \%, 40 \%$ and $30 \% \mathrm{MVC}$ in $\mathrm{CW}$ method were about $14 \mathrm{~s}, 31 \mathrm{~s}, 60$ $\mathrm{s}$, and $89 \mathrm{~s}$, respectively. Final force and last $30 \mathrm{~s}$ force in the PW method were about 27\% MVC and 35\% MVC, respectively. The $120 \mathrm{~s}$ force and final force in the $\mathrm{CW}$ method were 20 and $23 \%$ MVC, respectively.

\section{DISCUSSION}

High correlations (0.713-0.895) in this study were found among peak force, peak force time, final force, and last $30 \mathrm{~s}$ force. The peak force is an unsustainable progressive workload time point, and peak force time was $83.4 \mathrm{~s}$. Because these parameters evaluate muscle endurance after $1 \mathrm{~min}$ (80-120 s), they are considered to have high correlations among their parameters.

Meanwhile, parameters in the $\mathrm{CW}$ method showed high correlations $(r>0.70)$ only between respective nearness decreased times as follows: time to $80 \% \mathrm{MVC}$ and time to $60 \% \mathrm{MVC}$, time to $60 \% \mathrm{MVC}$ and time to $40 \%$ MVC, and time to $40 \% \mathrm{MVC}$ and time to $30 \% \mathrm{MVC}$. Since the decrease to $40 \%$ MVC takes about $1 \mathrm{~min}$, subjects feel significant pain for a long period of time. Meanwhile, the mean time of the peak force in the PW method, which gradually increases loads, was about $83 \mathrm{~s}$ without large pain sensation. This time is nearly equivalent to the time to $30 \% \mathrm{MVC}(88.8 \mathrm{~s})$ in the $\mathrm{CW}$ method, but the force $(63.9 \% \mathrm{MVC})$ was around twice that measured at $30 \% \mathrm{MVC}$. The forces exerted at $120 \mathrm{~s}$ in the CW and PW methods were at the $20 \%$ MVC level (PW: about 27\% MVC, CW: 23\% MVC). Hence, the PW method may be able to exert twice the force of the $\mathrm{CW}$ method at about $90 \mathrm{~s}$ without large pain sensation. However, the force after the peak force decreases remarkably similarly to the initial phase in the $\mathrm{CW}$ method, and the force after $120 \mathrm{~s}$ is considered to decrease to a nearly comparable level. Yamaji et al. [9] reported that the gripping force that a person can sustain without decrease was $15 \%$ MVC (about 150 s) of maximal grip. Hence, the exertion force in the PW method also reaches an almost steady state after $120 \mathrm{~s}$.

Peak force in the PW method correlated significantly with time to $40 \% \mathrm{MVC}$ in the $\mathrm{CW}$ method $(\mathrm{r}=0.605)$. The time to $40 \%$ MVC corresponds to the phase which 
Table 1. Correlations between PW parameters and CW parameters.

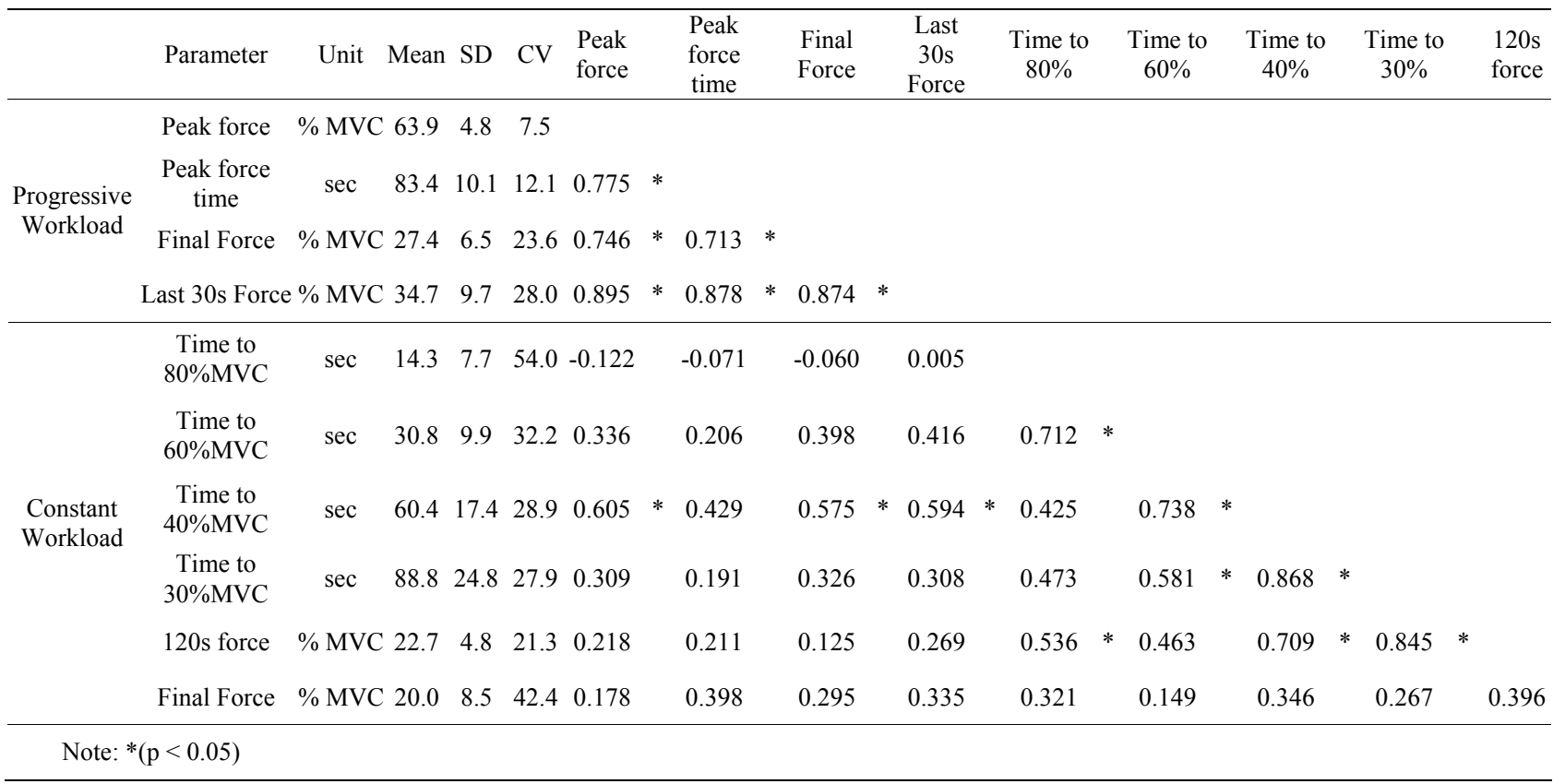

uses more oxygen due to blood flow reflux and which shows a gender difference in muscle endurance [16]. In short, this time is considered to evaluate the moving phase from a state of blood flow obstruction to a state of blood flow reflux. Peak force in the PW method is considered to closely relate with the above phase during maximal sustained gripping. From significant correlations $(\mathrm{r}=0.575-0.594)$ found between the time to $40 \%$ $\mathrm{MVC}$ in the CW method and the final force and last $30 \mathrm{~s}$ force in the PW method, the phase after reaching peak force may produce a similar phenomenon to the moving phase in the CW method.

Meanwhile, at a submaximal constant workload (ex. $50 \%$ MVC), the pain sensation may be reduced. However, according to Nagasawa et al. [6], the sustained time becomes very long when using a light workload $(50 \%$ MVC) which increases the sense of fatigue. Therefore, even a submaximal constant workload method cannot solve the problem.

Royce [17] compared force-decrease curves during sustained static maximal gripping with and without occlusion of arterial blood flow, reporting that they were similar in the phase of over $60 \%$ MVC. That is, it is considered that the phase over $60 \% \mathrm{MVC}$ in sustained static maximal gripping produces a blood flow obstruction and that the recruitment and fatigue of fast twitch fibers is largely reflected. Parameters evaluating the blood flow obstruction phase of sustained static maximal gripping in this study are time to $80 \% \mathrm{MVC}$ and time to $60 \%$ MVC. They may estimate an individual difference of muscle endurance in blood flow obstruction phase.
Significant correlations were not found between the above initial parameters (time to $80 \%$, time to $60 \% \mathrm{MVC}$ ) and 4 PW parameters. The PW parameters may not be able to evaluate the blood flow obstruction state.

However, in the case of the CW method by MVC, both phases of blood flow obstruction and blood flow reflux appear. Hence, Yamaji et al. [9] suggested that the $\mathrm{CW}$ method can evaluate muscle endurance in these two phases. In the future, the examination of the muscle oxygenation kinetics during force exertion by the PW method will be needed to clarify relationships with physiological parameters.

\section{SUMMARY}

In summary, peak force, final force, and last $30 \mathrm{~s}$ force in the PW method evaluate muscle endurance after 1 min (80-120 s), and they have high relationships. Significant relationships were found between time to $40 \%$ MVC in the CW method and peak force, final force, and last $30 \mathrm{~s}$ force in the PW method. The peak force in the PW method may be useful in that it is able to evaluate muscle endurance over a shorter time and without high pain sensation.

\section{REFERENCES}

[1] Clarke, D.H., Hunt, M.Q. and Dotson, C.H. (1992) Muscular strength and endurance as a function of age and activity level. Research Quarterly for Exercise and Sport, 63(3), 302-312. 
[2] Huczel, H.A. and Clarke, D.H. (1992) A comparison strength and muscle endurance in strength-trained and untrained women. European Journal of Applied Physiology and Occupational Physiology, 64(5), 467-470.

[3] Laforest, S., St-Pierre, D.M., Cyr, J. and Gayton, D. (1990) Effect of age and regular exercise on muscle strength and endurance. European Journal of Applied Physiology and Occupational Physiology, 60(2), 104-111.

[4] Larsson, B., Karlsson, S., Eriksson, M. and Gerdle, B. (2003) Test-retest reliability of EMG and peak torque during repetitive maximum concentric knee extensions. Journal of Electromyography and Kinesiology, 13(3), 281-287.

[5] Maughan, R.J., Harmon, M., Leiper, J.B., Sale, D. and Delman, A. (1986) Endurance capacity of untrained males and females in isometric and dynamic muscular contractions. European Journal of Applied Physiology and Occupational Physiology, 55(4), 395-400.

[6] Nagasawa, Y., Demura, S., Yoshimura, Y., Yamaji, S., Nakada, M. and Matsuzawa, J. (2000) Relationship between strength exertion and subjective muscle-fatigue sensation in the relative sustained static hand gripping. Japanese Journal of Physical Fitness and Sports Medicine, 49(4), 495-502.

[7] Nakada, M., Demura, S., Yamaji, S. and Nagasawa, Y. (2005) Examination of the reproducibility of grip force and muscle oxygenation kinetics on maximal repeated rhythmic grip exertion. Journal of Physiological Anthropology and Applied Human Science, 24(1), 1-6.

[8] Yamaji, S., Demura, S., Nagasawa, Y. and Nakada, M. (2004) Relationships between decreasing force and muscle oxygenation kinetics during sustained static gripping. Journal of Physiological Anthropology and Applied Human Science, 23(2), 41-47.

[9] Yamaji, S., Demura, S., Nagasawa, Y., Nakada, M., Yoshimura, Y., Matsuzawa, Z. and Toyoshima, Y. (2000) Examination of the parameters of static muscle endur- ance on sustained static maximal hand gripping. Japan Journal of Physical Education, 45(6), 695-706.

[10] Fitchett, M.A. (1985) Predictability of Vo2max from submaximal cycle ergometer and bench stepping tests. British Journal of Sports Medicine, 19(2), 85-88.

[11] Okura, T., Ueno, L.M. and Tanaka, K. (1998) Evaluation of cardiorespiratory fitness by submaximal graded cycling test using ratings of perceived exertion in Japanese young men. Japan Journal of Physical Education, 43(2), 102-116.

[12] Nakada, M., Demura, S., Yamaji, S., Minami, M., Kitabayashi, T. and Nagasawa, Y. (2004) Relationships between force curves and muscle oxygenation kinetics during repeated handgrip. Journal of Physiological Anthropology and Applied Human Science, 23(6), 191-196.

[13] West, W., Hicks, A., Clements, L. and Dowling, J. (1995) The relationship between voluntary electromyogram, endurance time and intensity of effort in isometric handgrip exercise. European Journal of Applied Physiology and Occupational Physiology, 71(4), 301-305.

[14] Yamaji, S., Demura, S., Nagasawa, Y., Nakada, M. and Kitabayashi, T. (2002) The effect of measurement time when evaluating static muscle endurance during sustained static maximal gripping. Journal of Physiological Anthropology and Applied Human Science, 21(3), 151158.

[15] Oldfield, R.C. (1971) The assessment and analysis of handedness: the Edinburgh inventory. Neuropsychologia, 9(1), 97-113.

[16] Yamaji, S., Demura, S. and Nakada, M. (2006) Sex differences and properties of the decreasing force during sustained static grip at various target forces. Perceptual and Motor Skills, 103(1), 29-39.

[17] Royce, J. (1958) Isometric fatigue curves in human muscle with normal and occluded circulation. The Research Quarterly, 29(2), 204-212. 